\title{
Investigations of short-term variations of vector and tensor anisotropies of cosmic rays using magnetic mirror model
}

\author{
P.Yu. Gololobov* and G.F. Krymsky and P.A. Krivoshapkin \\ Yu.G. Shafer insitute of cosmophysical research and aeronomy of SB RAS, Yakutsk, Russia \\ E-mail: gpetereikfia.sbras.ru, krymskyeikfia.sbras.ru, \\ p.a.krivoshapkin@mail.ru
}

\begin{abstract}
In the work variations of density, vector and tensor anisotropies of cosmic rays in the periods of Forbush decreases are investigated. The information about angular distribution of cosmic rays is obtained on the basis of the data of world-wide network of neutron monitors using the global survey method. Abrupt amplitude-phase oscillations of cosmic ray anisotropy during forbush decreases of unknown origin are found. In order to explain the obtained results the model of magnetic mirror is proposed. The model is based on kinetic consideration of the modulation process and allows to count the first two spherical harmonics of cosmic ray angular distribution. A comparison of the model calculations and the experimental data is carried out.
\end{abstract}

35th International Cosmic Ray Conference - ICRC2017

10-20 July, 2017

Bexco, Busan, Korea

\footnotetext{
* Speaker.
} 


\section{Introduction}

A flow of cosmic rays (CR) that is registered by ground-based detectors is characterized by the presence of isotropic and anisotropic components, the last one is observed by an individual $\mathrm{CR}$ detector as diurnal and semidiurnal variations which are originates due to the rotation of the Sun around its own axis. In average neutron monitors register diurnal variations with amplitude around $0.6 \%$ and phase $18 \mathrm{~h} \mathrm{LT}$ and semidiurnal variations with amplitude $0.1 \%$ and phase $3 \mathrm{~h} \mathrm{LT}$. But during periods of influence of different large-scale disturbances of solar wind the mentioned anistopy of CR can change greatly, moreover the characteristic time of influences of such structures on the CR flow may be from several days to hours. This moment makes strict requirements to a method of CR anisotropy measurements, first of all it is required the method that is able to provide sufficient time resolution.

In the current work the original method of global survey that was developed in ShICRA SB RAS is used. The method allows to determine the first two spherical harmonics of CR angular distribution. The first spherical harmonic consists of components: the first zonal harmonic $R_{1}^{0}$ and symmetric diurnal variation $R_{0}^{0}$ and can be represented in the space as a vector and because of that it is called a vector anisotropy. The second spherical harmonic consist of components: the second zonal harmonic $R_{2}^{0}$, antisymmetric diurnal $R_{2}^{1}$ and semidiurnal $R_{2}^{2}$ variations. This spherical harmonic can be represented in the space as a tensor ellipsoid and is called a tensor anisotropy.

Our latest investigations of the behavior of CR tensor anisotropy during the passage of large scale disturbances of solar wind $[1,2]$ have showed that during Forbush decreases this kind of anisotropy reveals very complicated behavior that is characterized by abrupt amplitude-phase oscillations which are difficult to explain. In this work the theoretical interpretation of such behavior is attempted.

\section{Model of CR modulation by magnetic mirror}

Forbush decreases of CR are caused by ejections of solar magnetized plasma. In case when the field in plasma is chaotic, the decrease is caused by scatterings of particles and their diffusion along an expanding volume. A regular magnetic field influences on CR with such way that a part of particle trajectories are forbidden. The case when the forbiddance is caused by a magnetic mirror was considered in [3]. Wherein it was supposed that the scatterings of particles in the field do not take place. It is of interest to consider a cooperation of the magnetic mirror and scattering of particles in a simple one-dimensional model.

The idealized formulation of problem suggests a homogeneous magnetic field $H$ that has compression of the field $H(x)$ as the magnetic mirror. It is exists a directional flow of CR that crosses the mirror along the field and undergoes scatterings, which are believed to be dotty and isotropic. The scattering centers are motionless in the reference system which are bounded to the mirror.

Let us write a function of CR distribution $f(x, \mu)$, where $x$ is the position of a particle relatively to the mirror and $\mu$ is a cosine of pitch angle. Since all the elements of magnetic field are motionless, the energy of particles does not change and we can track particles of a certain energy. Therefore the dependence on energy of particles does not included in the distribution function. 


$$
\frac{\partial f}{\partial t}=-\frac{\partial f}{\partial x} \dot{x}-\frac{\partial f}{\partial \mu} \dot{\mu}-\frac{1}{\tau}\left(f-\frac{1}{2} \int_{-1}^{1} f d \mu\right) .
$$

The first two terms in the right part of equation describe the influence of magnetic mirror subject to $\partial f / \partial t=0$ and the last term - the scatterings with a time constant $\tau$. The velocity of motion along the field $\dot{x}=v \mu$ and changes of pitch angle are regulated by the first adiabatic invariant $\left(1-\mu^{2}\right) / H(x)=$ const. Differentiating the last equation with respect to time and substituting $\dot{x}$, we obtain:

$$
\dot{\mu}=\frac{1-\mu^{2}}{2} \frac{H^{\prime}}{H} v .
$$

The distribution function will be presented by two spherical harmonics:

$$
f(x, \mu)=f^{(0)}(x)+f^{(1)}(x)+f^{(2)}(x)\left(\mu^{2}-1 / 3\right) .
$$

After substitution the distribution function into the equation for stationary case and emphasizing the zero, first and second harmonics, we would have the system of 3 equations:

$$
\begin{gathered}
\frac{\partial f^{(1)}}{\partial x}-\frac{H^{\prime}}{H} f^{(1)}=0 \\
\frac{\partial f^{(1)}}{\partial x}+\frac{4}{15} \frac{\partial f^{(2)}}{\partial x}-\frac{2}{5} \frac{H^{\prime}}{H} f^{(2)}+\frac{1}{v \tau}, f^{(1)}=0 \\
\frac{\partial f^{(1)}}{\partial x}-\frac{H^{\prime}}{2 H} f^{(1)}+\frac{1}{v \tau} f^{(2)}=0 .
\end{gathered}
$$

After integration we have:

$$
\begin{gathered}
f^{(1)}=\frac{H}{H_{0}} f_{0}^{(1)}, \\
f^{(2)}=-\frac{3}{2} v \tau \frac{H^{\prime}}{H_{0}} f_{0}^{(1)},
\end{gathered}
$$

where the index "1" marks the values for $x \rightarrow \infty$.

A behavior of isotropic intensity is determined by integrals:

$$
f^{(0)}=\frac{f^{(0)}}{H_{0}}\left[-\int \frac{H}{v \tau}-\frac{2}{5} \int\left(\frac{\left(H^{\prime}\right)^{2}}{H}-H^{\prime \prime}\right) v \tau d x\right],
$$

The divergence of the first integral in the upper and lower limits is associated with the simplified formulation of the problem. Actually the integration range is always limited. The second integral shows modulation effect of the mirror which is more greater, the more $\tau$ is, i.e. when the scatterings are few.

The obtained expression for $f^{(2)}$ indicates to the fact that during passage of the idealized magnetic mirror the second spherical harmonic (tensor anisotropy) have to experience a change of direction to the opposite one, while the first spherical harmonic (vector anisotropy) $f^{(1)}$ keeps its initial direction. The amplitudes of both kind of anisotropies can change with respect to compression of the magnetic field. 


\section{Comparison of magnetic mirror model with experimental data}

As was mentioned before, entering of the Earth into the area of influence of the magnetic mirror have to be characterized by abrupt amplitude-phase oscillations of the tensor anisotropy of $\mathrm{CR}$. Moreover, depending on the magnetic mirror parameters, the change of phase of at least one of the components of tensor anisotropy $R_{1}^{1}, R_{2}^{1}$ or $R_{2}^{2}$ to the opposite is expected.

In figure the behavior of components $R_{1}^{1}, R_{2}^{1}$ and $R_{2}^{2}$ that were obtained using the global survey method for the time period 5-9 May 2007 are shown as vectors on a hour dial. The behaviors of interplanetary magnetic field intensity $|B|$, proton density $\rho_{S W}$ and $v_{S W}$ of solar wind are also shown in the figure. AS seen from the figure at 7th of May an abrupt "jump" of all three solar wind parameters were observed. Which is originated due to the crossing of the neutral sheet by the Earth. As was mentioned in the works $[4,5]$ when the Earth crosses the neutral current sheet the magnetic mirror can be observed, which is originated in the interaction region of fast and slow flows of solar wind and accompanied by increases of solar wind pressure and field intensity. We can make a consequence that during such transition the behavior of tensor anisotropy that is expected from the magnetic mirror model have to be observed. Actually, during the crossing the short-term flip of the directions of components $R_{2}^{1}$ and $R_{2}^{2}$ are observed, after which they recover their initial direction.

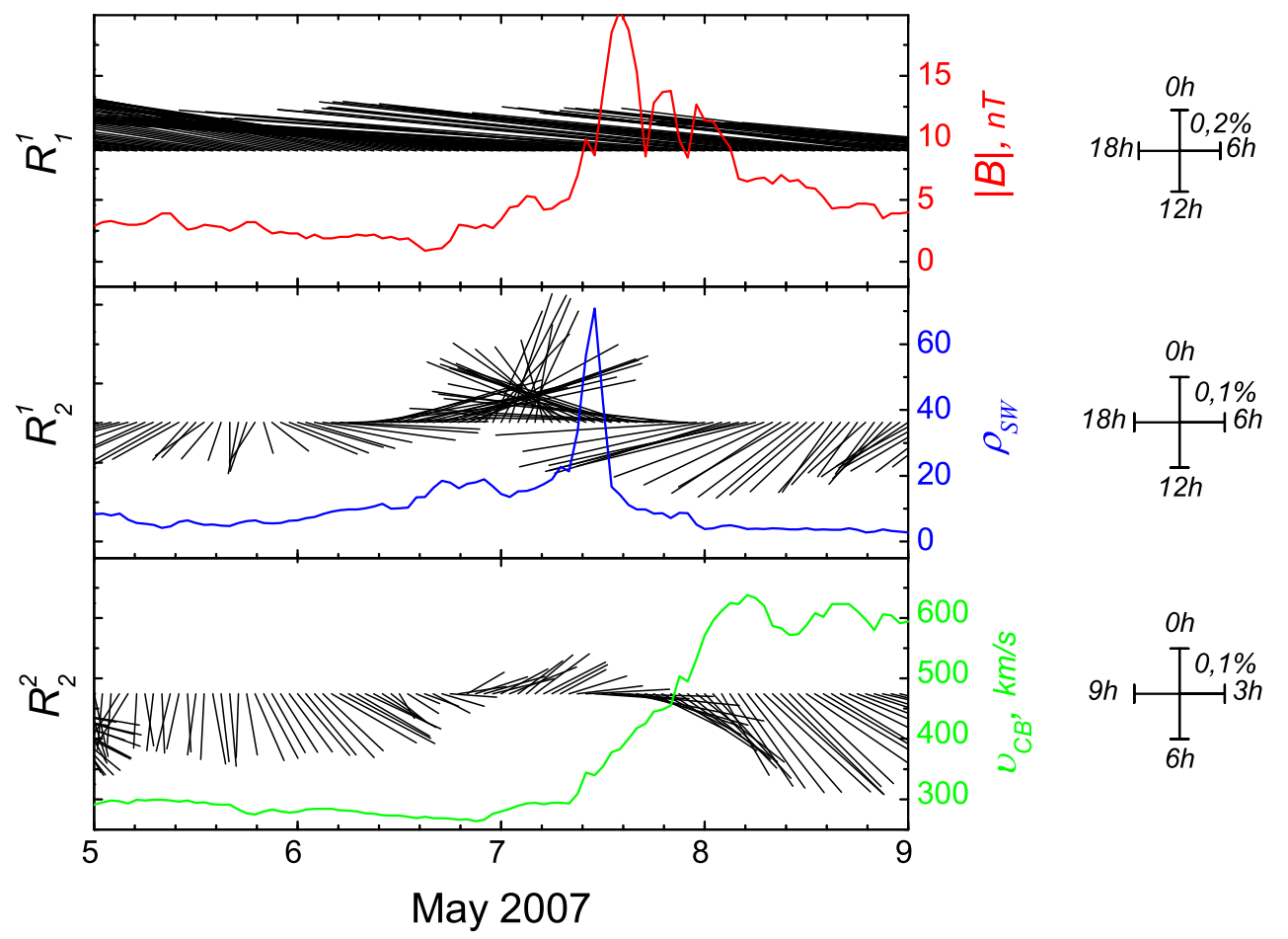

Figure 1: The behavior of $R_{1}^{1}, R_{2}^{1}, R_{2}^{2}$ and also the parameters of interplanetary magnetic field $|B|, \rho_{S W}, v_{S W}$ in May 2007. The components of anisotropy are shown as vectors on corresponding 24- or 12-hour dials, which are shown in the right part of the figure. 


\section{Conclusion}

The modeling of the behavior of vector and tensor anisotropies of CR during the transition through a magnetic mirror is conducted. It is established that during this moment the tensor anisotropy is expected to change its direction to the opposite one. Using of the global survey method by the data of world-wide network of neutron monitors the experimental data of angular distribution of $\mathrm{CR}$, which confirms this theory, were obtained.

\section{Acknowledgements}

The work was supported by the grants RFBR Nos. 15-42-05085-r_vostok_a, 15-42-05083-r_ vostok_a and the program of Presidium of SB RAS No. 23. The authors thank the World Data Center for Cosmic Rays for providing the data.

\section{References}

[1] S.K. Gerasimova, P.Yu. Gololobov, V.G. Grigoryev, P.A. Krivoshapkin, G.F. Krymsky, Investigation of short-term disturbances of the solar wind using a tensor anisotropy method, Proceedings of Science (2016) PoS(ICRC2015)077

[2] P.Yu. Gololobov, P.A. Krivoshapkin, G.F. Krymsky, V.G. Grigoryev, S.K. Gerasimova, Investigating tensor anisotropy of cosmic rays during large-scale solar wind disturbances, Solar-Terrestrial Physics 3 (2017) 22

[3] G.F. Krymsky, P.A. Krivoshapkin, V.P. Mamrukova, S.K. Gerasimova, Piston shock and forbush effect , Astronomy Letters 35 (2009) 696

[4] S.R. Thomas, M.J. Owens, M. Lockwood, C.J. Scott, Galactic cosmic ray modulation near the heliospheric current sheet, Solar Physics 289 (2014) 2653

[5] P.Yu. Gololobov, P.A. Krivoshapkin, G.F. Krymsky, V.G. Grigoryev, S.K. Gerasimova, Distribution of tensor anisotropy of cosmic rays near the neutral current sheet, Solar-Terrestrial Physics 3 (2017) 18 